\title{
Determination of LOOK ANGLes to Geostationary Communication Satellites
}

\author{
By Tomás Soler, ${ }^{1}$ Member, ASCE, and David W. Eisemann ${ }^{2}$
}

\begin{abstract}
Basic geodetic theory is applied to determine the geodetic azimuth and geodetic altitude required to point dish antennas to geostationary communication satellites. The mathematical treatment presented here takes into consideration the ellipticity of the earth. This generalization contrasts with standard formulas published in technical books in satellite communication engineering where a spherical approximation is implemented. Comparisons between the spherical and more rigorous ellipsoidal methods are discussed. Although the differences between the two approaches are not significant, they should be taken into consideration when very precise pointing to geostationary communication satellites or other space objects is dictated. The suggested method is simple to understand and straightforward to implement, and due to its advantages should replace any spherical alternative currently in usc.
\end{abstract}

\section{INTRODUCTION}

A practical and very common problem is the terrestrial pointing of earthbased parabolic antennas to satellites (e.g., geostationary communication satellites) and extragalactic radio sources [e.g., very long baseline interferometry (VLBI)]. Greater aiming accuracy is required for the exact pointing of optical instruments to stars or, using narrow-beam-laser ranging devices, pointing them to retroreflectors in orbiting space platforms. In all these cases the presumption is made that the earth-based tracking instruments have an altazimuth mounting. Therefore, the primary objective is to determine the spatial-object azimuth and altitude (for definitions see e.g., Mueller 1969 , p. 33) at some instant $t$. These two parameters are the socalled look angles in the terminology popularized by scientists and engineers specializing in the electrical/electronic field.

A plethora of recently published textbooks addressed to students of electronic engineering [e.g., Agraval (1986); Ha (1986); Pratt and Bostian (1986); Pattan (1993)] treat this subject along the same premises using a spherical approximation that assumes familiarity with spherical trigonometry. A more rigorous and comprehensive approach is introduced here based on elementary concepts of ellipsoidal geodesy and straightforward definitions of coordinate systems. No solution of spherical triangles is required in this latter implementation. The choice advocated in the present paper originates conceptually from classical equations in three-dimensional geodesy where the terrestrial target has been substituted by an artificial geostationary satellite.

Before the advent of digital satellite communications, most terrestrial communication links were established via ground-based communication lines. Beginning with the launch of the first commercial satellite, Early Bird, global

\footnotetext{
'Natl. Geodetic Survey, Coast and Geodetic Survey, Natl. Oc. Service, NOAA, 1315 East-West Highway, Silver Spring, MD 20910.

'Hughes Information \& Technology Co., 1768 Business Center Dr., Reston, VA 22090 .

Note. Discussion open until January 1, 1995. To extend the closing date one month. a written request must be filed with the ASCE Manager of Journals. The manuscript for this paper was submitted for review and possible publication on December 3, 1993. This paper is part of the Journal of Surveying Engineering, Vol. 120, No. 3, August, 1994. $\mathbb{C}$ ASCE, ISSN 0733-9453/94/0003-0115/ $\$ 2.00+\$ .25$ per page. Paper No. 7382.
} 
communication technology has changed at an unanticipated pace. All international, and almost all domestic long-distance television-program distribution is now established through spatial satellite connections. An increasing amount of international telephone traffic as well as all types of domestic data and voice communications are now transmitted through satellites instead of the standard optical-fiber links or line-of-sight microwave terrestrial networks.

To communicate with a satellite, ground-based reflector (dish) antennas are used. Reflector parabolic antennas can focus the transmitted power from/to a narrow region of the sky. This allows for establishment of communication links over long distances, minimizing transmitted electromagnetic power requirements. However, because the signal is concentrated in a narrow region of the sky, the antenna must be precisely pointed at the emitting/receiving source. The problems in pointing an antenna can range from simple to complex, depending on the motion of the satellite in its orbit.

Most communication satellites use circular geostationary orbits. In this particular situation, the satellite remains above a fixed location on the earth's equator at a constant geocentric distance. This greatly facilitates pointing at the satellite from a ground-based antenna when the satellite is above the observer horizon. Aside from data transfer to/from satellites, dish antennas are typically used in radio astronomy for studying extraterrestrial radio sources. The problem of pointing toward a celestial object, as opposed to a geostationary communication satellite, is complicated by the earth's rotation. The apparent position of the object on the celestial sphere constantly changes with time and, among others, effects of precession, nutation, and polar motion must be taken into consideration. This case is beyond the scope of the present work and is not discussed.

Similarly, the conversion from geodetic azimuth and vertical angle to "true" astronomic azimuth and altitude is left outside this presentation because of the difficulties associated with accurately knowing the components of the deflection of the vertical at the point where the antenna or tracking instrument is located.

\section{SPHERICAL APPROXIMATION}

The place on the earth's surface where the dish antenna is located is denoted by $\mathrm{P}^{\prime}$ (see Fig. 1). Assume that its position has spherical coordinates $\left(\lambda, \phi^{\prime}\right)$ where $\lambda$ always denotes longitude, measured positive east, and $\phi^{\prime}$ indicates spherical (geocentric) latitude. Assume furthermore that the subsatellite point $\mathrm{E}$-intersection with the earth's surface of the geocentric radius vector to the satellite $S-$ is on the equator at a longitude $\lambda_{5}$. The angle $\gamma$ between the radius vectors of points $\mathrm{P}^{\prime}$ and $\mathrm{E}$ can be obtained using the right spherical triangle $P^{\prime} Q E$ depicted in Fig. 1. Applying Napier's rules it follows that

$$
\cos \gamma=\cos \phi^{\prime} \cos \left(\lambda_{\mathrm{s}}-\lambda\right)
$$

The distances OP' and OS, respectively $R$ and $r$, are related with the angle $\gamma$ by the equation (see Fig. 1)

$$
d=r\left[1+(R / r)^{2}-2(R / r) \cos \gamma\right]^{1 / 2}
$$

where $d=$ topocentric distance from the antenna to the satellite, or alternatively, the range of object from observer; $r$ is used to denote the geocentric distance from the earth's center to the spacecraft, which for an ideal geo- 


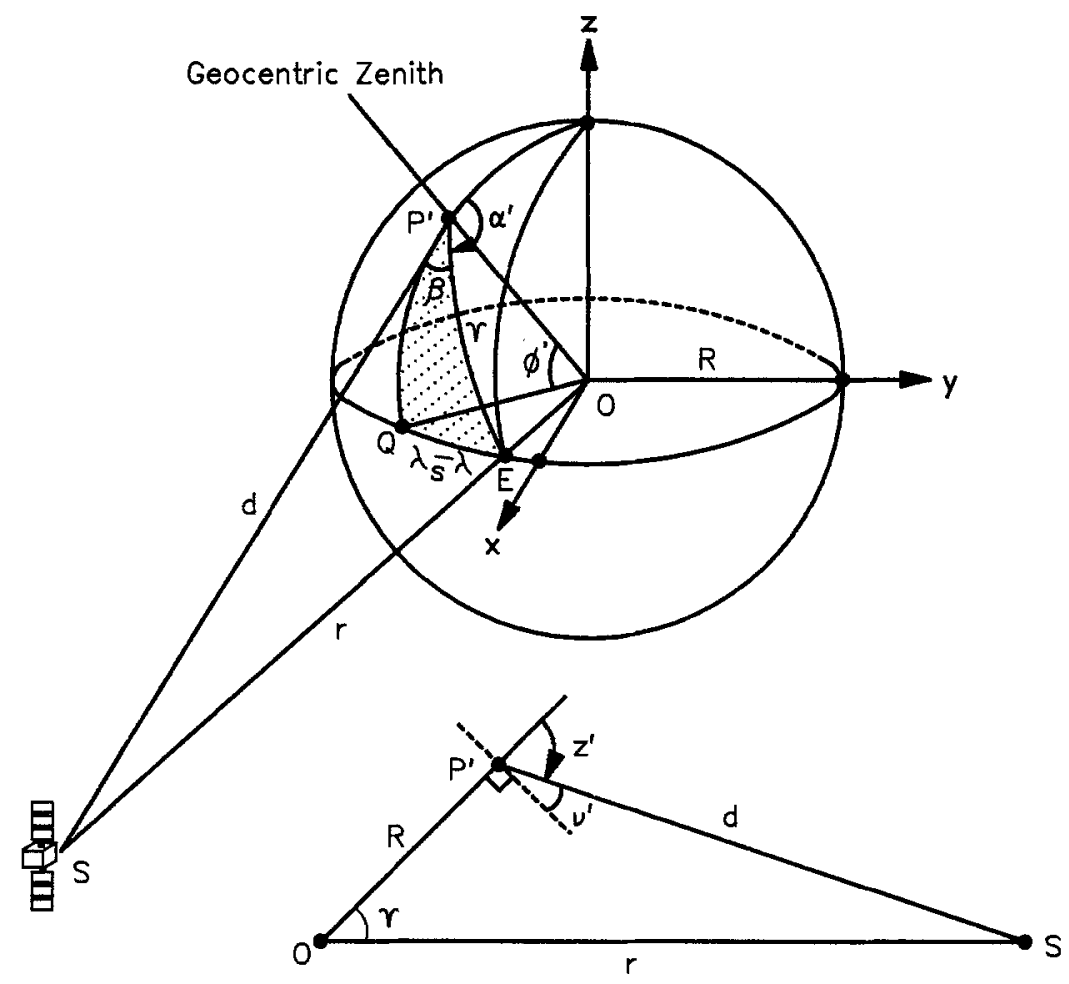

FIG. 1. Look Angles Calculation Assuming Spherical Earth

stationary satellite is constant, $r \simeq 42,200 \mathrm{~km}$; and $R=$ a "mean value" for the radius of the earth; the radius of a sphere that has the same volume as the earth ellipsoid; hence, $R=6,371 \mathrm{~km}$ (Moritz 1992).

Using the law of sines it can be written

$$
r / \sin z^{\prime}=d / \sin \gamma
$$

from where the spherical (geocentric) zenith distance $z^{\prime}$ of satellite $S$ at antenna location $\mathrm{P}^{\prime}$ can be solved for

$$
z^{\prime}=\sin ^{-1}[(r / d) \sin \gamma]
$$

From this, the altitude $\gamma^{\prime}$ follows immediately: Because the term altitude specifically refers to the local astronomic horizon, a more appropriate word would be spherical vertical angle; thus, if $v^{\prime}=90^{\circ}-z^{\prime}$, then

$$
v^{\prime}=\cos ^{-1}[(r / d) \sin \gamma]
$$

From Fig. 1 it is clear that the azimuth $\alpha^{\prime}$ of the satellite is a function of the angle $\beta$. In this particular situation, as can be seen in the Fig. $1, \alpha^{\prime}=$ $180^{\circ}-\beta$.

The angle $\beta$ is computed using Napier's rules as

$$
\beta=\cos ^{-1}\left(\cot \gamma \tan \phi^{\prime}\right)
$$

Consequently, once the vertex angle $\beta$ at $\mathrm{P}^{\prime}$ is known, the value of the 
azimuth is easily determined using one of the four possible scenarios shown in Fig. 2.

\section{RIGOROUS ELLIPSOIDAL APPROACH}

To improve the accuracy of the calculation of the look angles, the spherical approximation of the earth is replaced by an oblate ellipsoid of revolution. This approach eliminates errors introduced by spherical geometry and, consequently, discrepancies due to differences in definition between geocentric and geodetic parameters (i.e., zenith distances and latitudes). Fig. 3 illustrates the advantage of using an ellipsoidal model of the earth. The value of the geodetic zenith distance $z$ (referred to the normal to the ellipsoid) is more accurate than $z^{\prime}$ because the location of the observer at point $\mathbf{P}$ is closer to its true location on the earth surface by the mere fact that the earth more accurately resembles a flattened ellipsoid than a sphere. In the
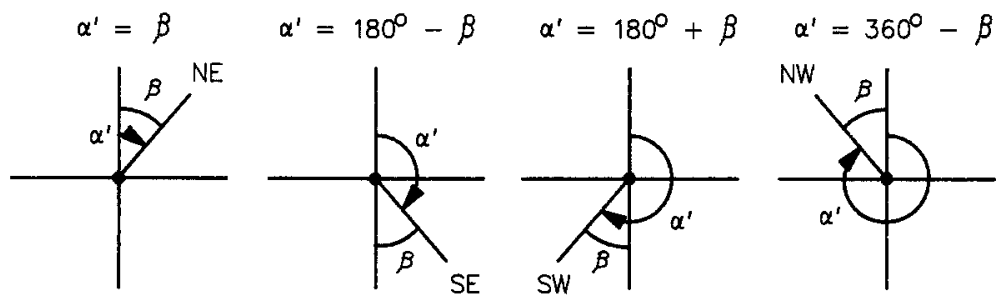

FIG. 2. Azimuth Calculation as Function of Subsatellite Point Location with Respect to Earth Station

\section{FIGURE NOT TO SCALE}

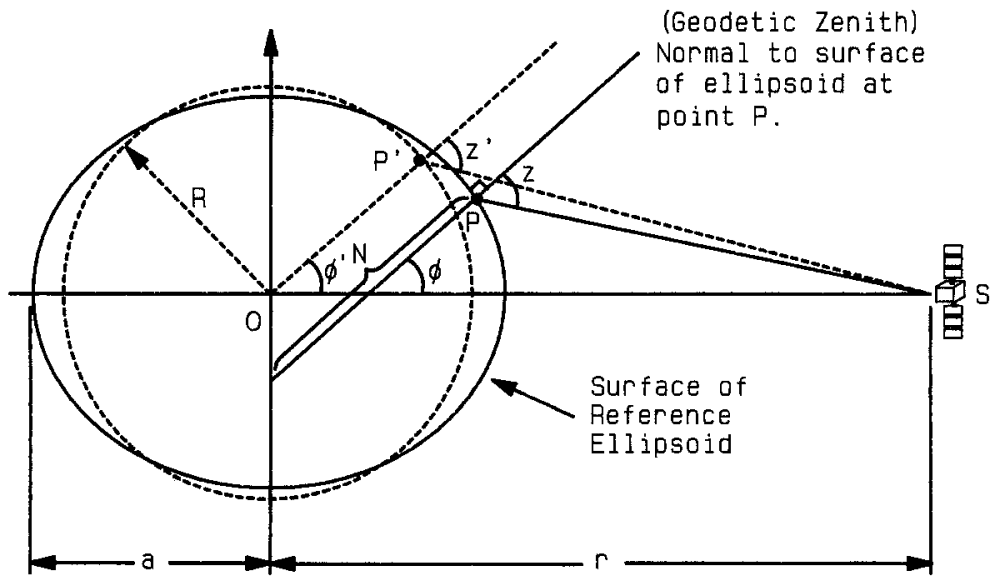

$$
\begin{aligned}
& \left.\begin{array}{l}
\phi=\text { Geodetic Latitude of } P \\
\phi^{\prime}=\text { Geocentric Latitude of } P^{\prime}
\end{array}\right\} \phi=\phi^{\prime} \\
& z=\text { Zenith distance measured using ellipsiodal earth. } \\
& z^{\prime}=\text { Zenith distance measured using spherical earth. }
\end{aligned}
$$

FIG. 3. Spherical and Geodetic Zenith Distances 


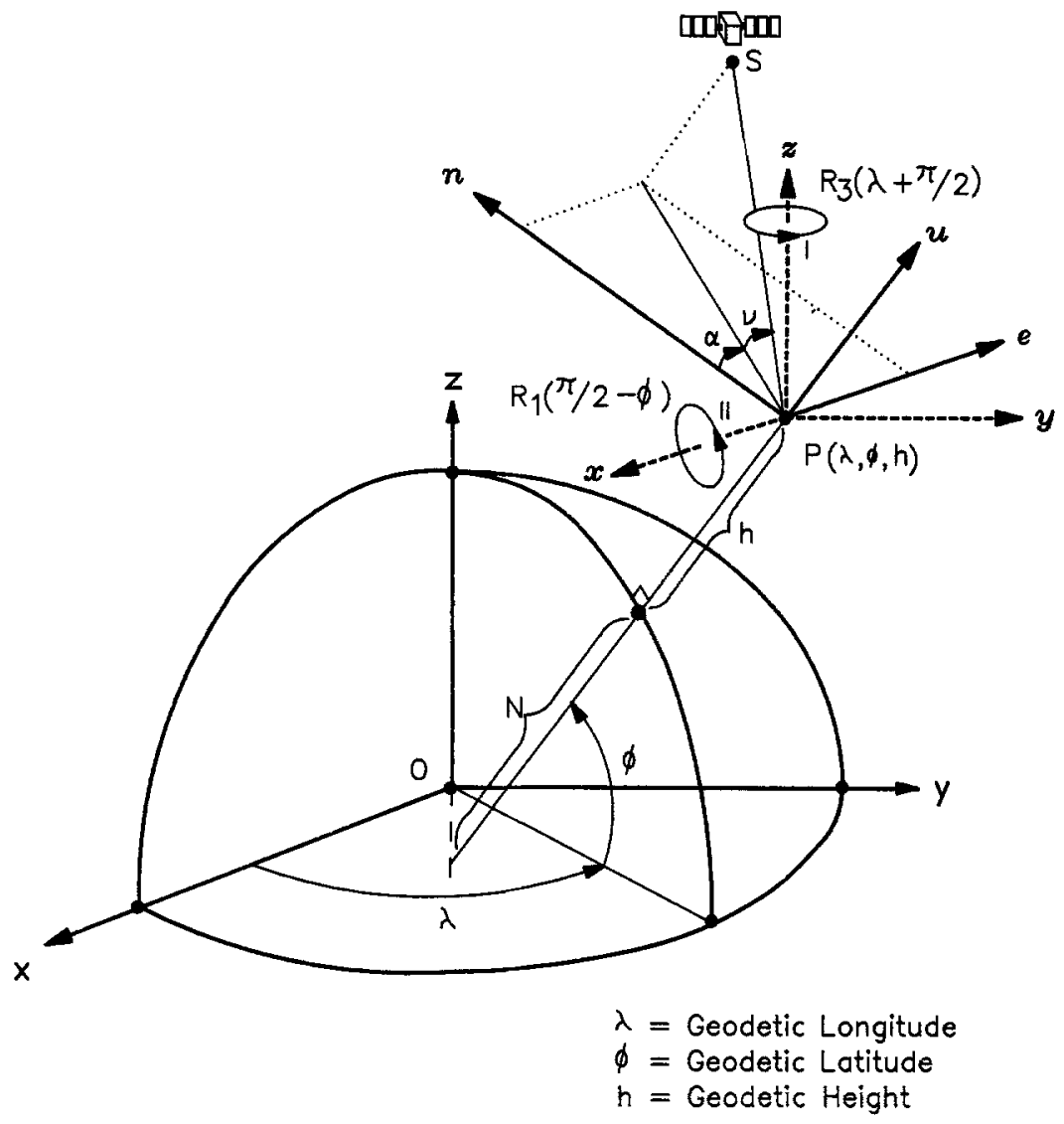

FIG. 4. Transformation From Local Terrestrial $(x, y, z)$ to Local Geodetic $(e, n, u)$

figure, we are assuming that, as usual, the geodetic latitude $\phi$, where the antenna is located, is known a priori. Note that if this value is interpreted as spherical latitude, an error in the value of the zenith distance is introduced.

The ellipsoid of revolution adopted in this investigation is the GRS 80 ellipsoid (Mortiz 1992). The two geometric parameters of interest are its size, defined by the semimajor axis, $a=6,378,137 \mathrm{~m}$, and its shape, specified by the flattening $f$ or, equivalently, its inverse, $f^{-1}=298.257222101$.

Therefore, it is critical, for sake of rigor, that all pertinent calculations be made with respect to the local geodetic coordinate system $(e, n, u)$ at $\mathrm{P}$ (see Fig. 4). There are several possible selections of coordinate axes (e.g., left-handed, as opposed to right-handed, the one preferred here) for such a local frame [e.g., Rapp (1979); Leick (1990); Burkholder (1993)]. The definitions and nomenclature in the present study follow closely those in Soler and Hothem $(1988,1989)$.

The geodetic azimuth $\alpha$ and "geodetic altitude" $v$ (i.e., geodetic vertical angle) of the satellite can be computed using

$$
\tan \alpha=e / n
$$




$$
\tan v=u /\left(e^{2}+n^{2}\right)^{1 / 2}
$$

This implies that the components of the topocentric range PS to the satellite in the $(e, n, u)$ local geodetic coordinate system are required. These can be calculated by transforming the components along the $(x, y, z)$ frame at $\mathrm{P}$, into the $(e, n, u)$ system. The $(x, y, z)$ frame is parallel to the geocentric conventional terrestrial reference frame $(\mathbf{x}, \mathbf{y}, \mathbf{z})$. This transformation is performed applying the rotation matrix $[R]$ defined as [e.g., Soler (1976)]

$$
\begin{gathered}
{[R]=R_{1}(1 / 2 \pi-\phi) R_{3}(\lambda+1 / 2 \pi)} \\
{[R]=\left[\begin{array}{ccc}
-\sin \lambda & \cos \lambda & 0 \\
-\sin \phi \cos \lambda & -\sin \phi \sin \lambda & \cos \phi \\
\cos \phi \cos \lambda & \cos \phi \sin \lambda & \sin \phi
\end{array}\right]}
\end{gathered}
$$

and the matrix equation

$$
\left\{\begin{array}{l}
e \\
n \\
u
\end{array}\right\}=[R]\left\{\begin{array}{l}
x \\
y \\
z
\end{array}\right\}
$$

It follows immediately that the coordinates of satellite $\mathrm{S}$ along the $(x, y, z)$ local coordinate system are

$$
\left\{\begin{array}{l}
x \\
y \\
z
\end{array}\right\}=\left\{\begin{array}{l}
\mathbf{x}_{\mathrm{S}} \\
\mathbf{y}_{\mathrm{S}} \\
\mathbf{z}_{\mathrm{S}}
\end{array}\right\}-\left\{\begin{array}{l}
\mathbf{x}_{\mathrm{P}} \\
\mathbf{y}_{\mathrm{P}} \\
\mathbf{z}_{\mathrm{P}}
\end{array}\right\}
$$

The rectangular coordinates $\mathbf{x}, \mathbf{y}, \mathbf{z}$ of satellite $S$ and antenna location $P$.can be readily computed from their known curvilinear geodetic coordinates using well-known expressions

$$
\left\{\begin{array}{l}
\mathbf{x} \\
\mathbf{y} \\
\mathbf{z}
\end{array}\right\}=\left\{\begin{array}{l}
(N+h) \cos \phi \cos \lambda \\
(N+h) \cos \phi \sin \lambda \\
{\left[N\left(1-e^{2}\right)+h\right] \sin \phi}
\end{array}\right\}
$$

where $N$ (see Figs. 3 and 4 ) is the principal radius of curvature in the prime vertical, $N=a / W$ with $W=\left(1-e^{2} \sin ^{2} \phi\right)^{1 / 2}$; and the square of the ellipsoid eccentricity is given in closed form by $e^{2}=2 f-f^{2}$.

The derivation advanced herein is general and applies to any type of earth-orbiting artificial satellite. The position of the satellite in rectangular coordinates $(\mathbf{x}, \mathbf{y}, \mathbf{z})_{\mathrm{s}}$ may be directly available from its ephemeris given at constant time intervals, or, alternatively, they can be computed from the satellite's six orbital Keplerian elements using standard procedures [e.g., Leick (1990)]. It should be realized that getting these quantities in real time is a problem in itself and generally one would rely on predicted values.

The curvilinear geodetic coordinates for the antenna location were implicitly given above as $(\lambda, \phi, h)$ where $h$ is the ellipsoidal (geodetic) height of the point. If necessary, this parameter can be approximated by $h \simeq N_{g}$ $+H$, where $N_{g}$ is the undulation of the geoid or geoid height, and $H$ is the orthometric height, also loosely referred to as elevation or mean sea-level height. $H$ can be accurately determined through geodetic leveling or approximately interpolated from topographic maps. The value of $N_{g}$ requires access to modern computer software that is able to numerically model the geoid and estimate geoid heights. In the conterminous United States, $N_{g}$ is approximately bounded by the values $-35 \mathrm{~m}<N_{g}<-7 \mathrm{~m}$. 
In the special case of an ideal geostationary communication satellite $\mathrm{S}$ moving in a circular orbit of a period $T$ of a sidereal day and coplanar with the earth's equator $\left(e_{\mathrm{s}}=0 ; i_{\mathrm{s}}=0^{\circ} ; T=24 h\right)$, its position in a curvilinear coordinates is simplified accordingly: $\phi_{\mathrm{s}}=0^{\circ}$, and $h_{\mathrm{s}}$ is known, $h_{\mathrm{s}}=r-$ $a$ (see Fig. 3). Thus, in essence, the only needed variable is $\lambda_{\mathrm{s}}$, defining the location of the satellite on the equatorial plane.

Fig. 5 presents a self-explanatory step-by-step block diagram to implement the theory just described. Notice that as an added advantage of this approach, the final value of the azimuth is determined through the inverse of a tangent trigonometric function that has a fraction as an argument. Consequently, by using standard subroutines that are readily available in the libraries of most compilers (e.g., DATAN2), the exact quadrant where the

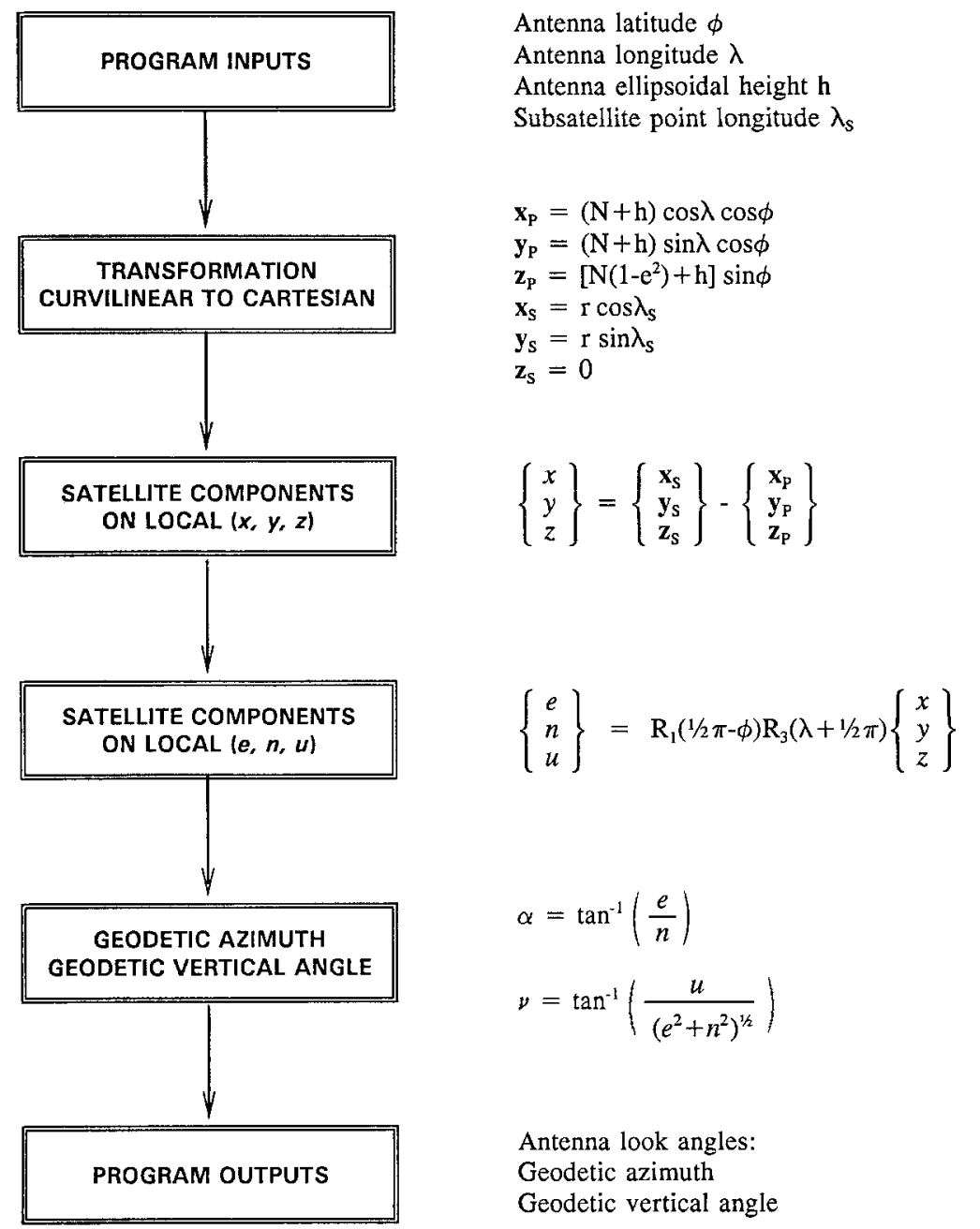

FIG. 5. Calculation of Look Angles to Geostationary Satellites Using Ellipsoidal Earth 
angle is located can be found without having to turn to sequential checks of the type depicted in Fig. 2. This algorithm should be helpful to any reader interested in applying the technique. A PC DOS compatible MS FORTRAN program is available from the writers on request. Simple computer programs such as this one makes obsolete the nomograms that are frequently reproduced in satellite-communication textbooks to visually interpolate azimuth and vertical angles.

\section{COMPARISON OF RESULTS}

To quantify the differences between the spherical and ellipsoidal methods, a set of numerical examples was analyzed. Instead of using a single location on the earth's surface, the distinctive symmetry of the problem was exploited to produce a tabulation from which any case could be interpreted. The reader is well aware that, presently, many geostationary satellites around the equator are active. For example, communication satellites visible at Washington, D.C., that broadcast popular TV channels include, among others, F2 (SATCOM 2R; $\lambda_{\mathrm{s}}=-72^{\circ}$ ); G5 (GALAXI $5 ; \lambda_{\mathrm{s}}=-125^{\circ}$ ); F3 $\left(\mathrm{SATCOM} \mathrm{C} 3 ; \lambda_{\mathrm{s}}=-131^{\circ}\right)$; and F1 (SATCOM C1; $\left.\lambda_{\mathrm{s}}=-157^{\circ}\right)$.

Initially, Table 1 was compiled. It shows the differences between the

TABLE 1. Caiculated Pointing Angles in Degrees to Geostationary Satellite $\left(\phi_{S}=0^{\circ}\right)$ for Different Earth-Station Latitudes under Condition $\lambda=\lambda_{S}$

\begin{tabular}{|c|c|c|c|c|c|c|}
\hline \multirow{2}{*}{$\begin{array}{c}\text { Earth-station } \\
\text { latitude } \\
(1)\end{array}$} & \multicolumn{2}{|c|}{$\begin{array}{c}\text { Pointing Angles } \\
\text { Assuming Spherical } \\
\text { Earth }\end{array}$} & \multicolumn{2}{|c|}{$\begin{array}{c}\text { Pointing Angles } \\
\text { Assuming Ellipsoidal } \\
\text { Earth }\end{array}$} & \multicolumn{2}{|c|}{$\begin{array}{c}\text { Difference } \\
\text { (Ellipsoidal-Spherical } \\
\text { Method) }\end{array}$} \\
\hline & $\begin{array}{c}\text { Azimuth } \\
\text { (2) }\end{array}$ & $\begin{array}{l}\text { Vertical angle } \\
\text { (3) }\end{array}$ & $\begin{array}{c}\text { Azimuth } \\
(4)\end{array}$ & $\begin{array}{c}\text { Vertical angle } \\
\text { (5) }\end{array}$ & $\begin{array}{c}\text { Azimuth } \\
(6)\end{array}$ & $\begin{array}{c}\text { Vertical angle } \\
(7)\end{array}$ \\
\hline 0 & 180 & 90.0000 & 180 & 90.0000 & 0 & 0 \\
\hline 5 & 180 & 84.1139 & 180 & 84.1185 & 0 & 0.0046 \\
\hline 10 & 180 & 78.2386 & 180 & 78.2475 & 0 & 0.0089 \\
\hline 15 & 180 & 72.3843 & 180 & 72.3972 & 0 & 0.0129 \\
\hline 20 & 180 & 66.5612 & 180 & 66.5775 & 0 & 0.0163 \\
\hline 25 & 180 & 60.7782 & 180 & 60.7972 & 0 & 0.0190 \\
\hline 30 & 180 & 55.0434 & 180 & 55.0645 & 0 & 0.0211 \\
\hline 35 & 180 & 49.3641 & 180 & 49.3864 & 0 & 0.0223 \\
\hline 40 & 180 & 43.7459 & 180 & 43.7688 & 0 & 0.0229 \\
\hline 42.98 & 180 & 40.4285 & 180 & 40.4515 & 0 & 0.0230 \\
\hline 45 & 180 & 38.1935 & 180 & 38.2164 & 0 & 0.0229 \\
\hline 50 & 180 & 32.7105 & 180 & 32.7329 & 0 & 0.0224 \\
\hline 55 & 180 & 27.2990 & 180 & 27.3207 & 0 & 0.0217 \\
\hline 60 & 180 & 21.9605 & 180 & 21.9811 & 0 & 0.0206 \\
\hline 65 & 180 & 16.6951 & 180 & 16.7147 & 0 & 0.0196 \\
\hline 70 & 180 & 11.5023 & 180 & 11.5210 & 0 & 0.0187 \\
\hline 75 & 180 & 6.3810 & 180 & 6.3989 & 0 & 0.0179 \\
\hline 80 & 180 & 1.3291 & 180 & 1.3467 & 0 & 0.0176 \\
\hline 81.326 & 180 & 0 & 180 & 0.0174 & 0 & 0.0174 \\
\hline 81.344 & - & - & 180 & 0 & - & - \\
\hline 85 & - & - & - & - & - & - \\
\hline 90 & 一 & - & - & - & - & - \\
\hline
\end{tabular}

Note: A missing value indicates that satellite is below the observer's horizon. 
spherical and ellipsoidal options assuming antennas located on the reference meridian $\left(\lambda=0^{\circ}\right)$ but at different positive geodetic latitudes separated by increments of $5^{\circ}$. Notice that in this table a single geostationary satellite located at $\lambda_{\mathrm{s}}=0^{\circ}$ is postulated.

The first important conclusion is that for observers above latitude $81^{\circ} .34$, all true geostationary satellites are always below the local horizon, and thus not visible. Hence, $-81^{\circ} .34<\phi<81^{\circ} .34$ determines the approximate range of latitude that an earth station must have in order to successfully communicate with a satellite parked in geostationary orbit. These latitude limits should not be taken literally, because small vertical angles (e.g., $\left.<10^{\circ}\right)$ are more sensitive to receiver noise due to atmospheric refraction, earth's thermal emission, line-of-sight obstructions, signal reflections with the ground or nearby structures, and other factors, sometimes making ground-satellite communications difficult or impractical.

To further visualize the tabulated values, Fig. 6 shows the error introduced in the vertical angle when the ellipsoidal approach is replaced by a spherical approximation. The maximum difference never reaches more than $0^{\circ} .025$ $=1^{\prime} .5$. Although this quantity may be considered insignificant, it should be stressed that a directional error of this magnitude at the antenna location translates into a position error of approximately $2.6 \mathrm{~km}$ at satellite height. Consequently, whereas this may not affect communication using wide-beam dish antennas, it should not be neglected in situations with highly directive gain antennas or when accurate directional pointing (e.g., by laser) is attempted.

The peculiar shape of the curve in Fig. 6 is a consequence of the geometric properties implicit in the definitions of spherical and geodetic (ellipsoidal) zenith distances. The maximum difference between the spherical and ellip-

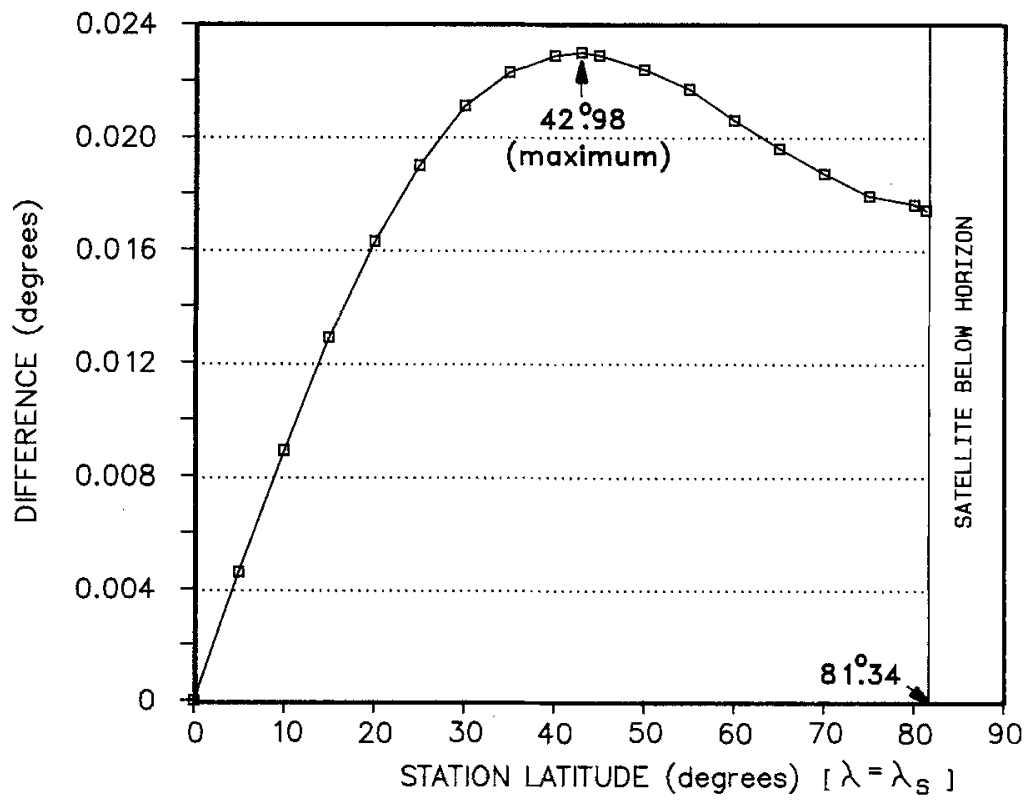

FIG. 6. Difference in Degrees in Calculated Elevation Pointing Angles versus Latitude of Observer (Ellipsoidal Method - Spherical Method) 
soidal approximations is $0^{\circ} .023$ reached at stations with latitude $\pm 42^{\circ} .98$ and then decreases to a value of $0^{\circ} .018$ when the satellite is on the local horizon plane at stations with latitude $\pm 81^{\circ} .34$. At higher (positive) and lower (negative) latitudes, geostationary satellites have negative vertical angles and are never above the horizon.

The results in Table 1 do not change when the antenna is located in the southern hemisphere, except that the azimuth to the satellite is $0^{\circ}$ instead of $180^{\circ}$. This is immediately explained by simple geometric arguments. Further, the tabulated differences and the same when longitudes for both the earth-station location are the subsatellite point have identical values, or in other words, when the meridians of the satellite and the antenna coincide.

A more complicated situation arises when antenna and satellite are not on the same meridian. Obviously, in this general case, not only the elevation angles but also the azimuth angles resulting from applying the spherical or ellipsoidal methods disagree.

Table 2 and Fig. 7 summarize the results assuming a station located at $\lambda$ $=0^{\circ}, \phi=45^{\circ}$. Notice that while the largest difference in elevation always occurs when the satellite is on the meridian of the antenna, the difference in azimuth increases with the value of $\lambda_{\mathrm{s}}-\lambda$, until it reaches a maxi$\mathrm{mum} /$ minimum of $\pm 0^{\circ} .029$ before the satellite disappears from view when its longitude is $-77^{\circ} .69<\lambda_{\mathrm{s}}<77^{\circ} .69$. This can be generalized for any other location. For example, at Washington, D.C. $\left(\lambda=-77^{\circ} .13 ; \phi=38^{\circ} .75\right)$, only geostationary satellites with theoretical limits $-154^{\circ} .82<\lambda_{\mathrm{s}}<0^{\circ} .54$ are visible.

Fig. 7 shows an interesting characteristic of the plotted differences: the magnitude of the error-in absolute sense-introduced in azimuth and vertical angle are inversely related. That is, a maximum error in azimuth

TABLE 2. Calculated Pointing Angles in Degrees with Geostationary Satellite $\left(\phi_{\mathrm{S}}=0^{\circ}\right)$ at Different Longitudes (Earth Station Assumed at $\lambda=0^{\circ}, \phi=45^{\circ}$ )

\begin{tabular}{|c|c|c|c|c|c|c|}
\hline \multirow{2}{*}{$\begin{array}{l}\text { Subsatellite- } \\
\text { point longitude } \\
\text { (1) }\end{array}$} & \multicolumn{2}{|c|}{$\begin{array}{l}\text { Pointing Angles Assuming } \\
\text { Spherical Earth }\end{array}$} & \multicolumn{2}{|c|}{$\begin{array}{l}\text { Pointing Angles Assuming } \\
\text { Ellipsoidal Earth }\end{array}$} & \multicolumn{2}{|c|}{$\begin{array}{l}\text { Difference (Ellipsoidal- } \\
\text { Spherical Method) }\end{array}$} \\
\hline & $\begin{array}{l}\text { Azimuth } \\
\text { (2) }\end{array}$ & $\begin{array}{l}\text { Vertical angle } \\
\text { (3) }\end{array}$ & $\begin{array}{l}\text { Azimuth } \\
\text { (4) }\end{array}$ & $\begin{array}{l}\text { Vertical angle } \\
\text { (5) }\end{array}$ & $\begin{array}{l}\text { Azimuth } \\
\text { (6) }\end{array}$ & $\begin{array}{l}\text { Vertical angle } \\
\text { (7) }\end{array}$ \\
\hline 0 & 180.0000 & 38.1935 & 180.0000 & 38.2164 & 0 & 0.0229 \\
\hline 10 & 165.9981 & 37.2411 & 165.9883 & 37.2629 & -0.0098 & 0.0218 \\
\hline 20 & 152.7637 & 34.5024 & 152.7459 & 34.5215 & -0.0178 & 0.0191 \\
\hline 30 & 140.7685 & 30.2785 & 140.7453 & 30.2941 & -0.0232 & 0.0156 \\
\hline 40 & 130.1207 & 24.9386 & 130.0943 & 24.9504 & -0.0264 & 0.0118 \\
\hline 50 & 120.6821 & 18.8282 & 120.6540 & 18.8367 & -0.0281 & 0.0085 \\
\hline 60 & 112.2077 & 12.2299 & 112.1789 & 12.2358 & -0.0288 & 0.0059 \\
\hline 70 & 104.4328 & 5.3605 & 104.4038 & 5.3646 & -0.0290 & 0.0041 \\
\hline 75 & 100.7286 & 1.8768 & 100.6996 & 1.8804 & -0.0290 & 0.0036 \\
\hline 77.6865 & 98.7743 & 0.0000 & 98.7453 & 0.0034 & -0.0290 & 0.0034 \\
\hline 77.6914 & - & - & 98.7418 & 0.0000 & - & - \\
\hline-10 & 194.0019 & 37.2411 & 194.0117 & 37.2629 & 0.0098 & 0.0218 \\
\hline-20 & 207.2363 & 34.5024 & 207.2541 & 34.5215 & 0.0178 & 0.0191 \\
\hline-30 & 219.2315 & 30.2785 & 219.2547 & 30.2941 & 0.0232 & 0.0156 \\
\hline-40 & 229.8792 & 24.9386 & 229.9057 & 24.9504 & 0.0265 & 0.0118 \\
\hline-50 & 239.3179 & 18.8282 & 239.3460 & 18.8367 & 0.0281 & 0.0085 \\
\hline-60 & 247.7923 & 12.2299 & 247.8211 & 12.2358 & 0.0288 & 0.0059 \\
\hline-70 & 255.5672 & 5.3605 & 255.5962 & 5.3646 & 0.0290 & 0.0041 \\
\hline-75 & 259.2714 & 1.8768 & 259.3004 & 1.8804 & 0.0290 & 0.0036 \\
\hline-77.6865 & 261.2257 & 0.0000 & 261.2547 & 0.0034 & 0.0290 & 0.0034 \\
\hline-77.6914 & - & - & 261.2582 & 0.0000 & - & - \\
\hline
\end{tabular}

Note: A missing value indicates that satellite is below the observer's horizon. 


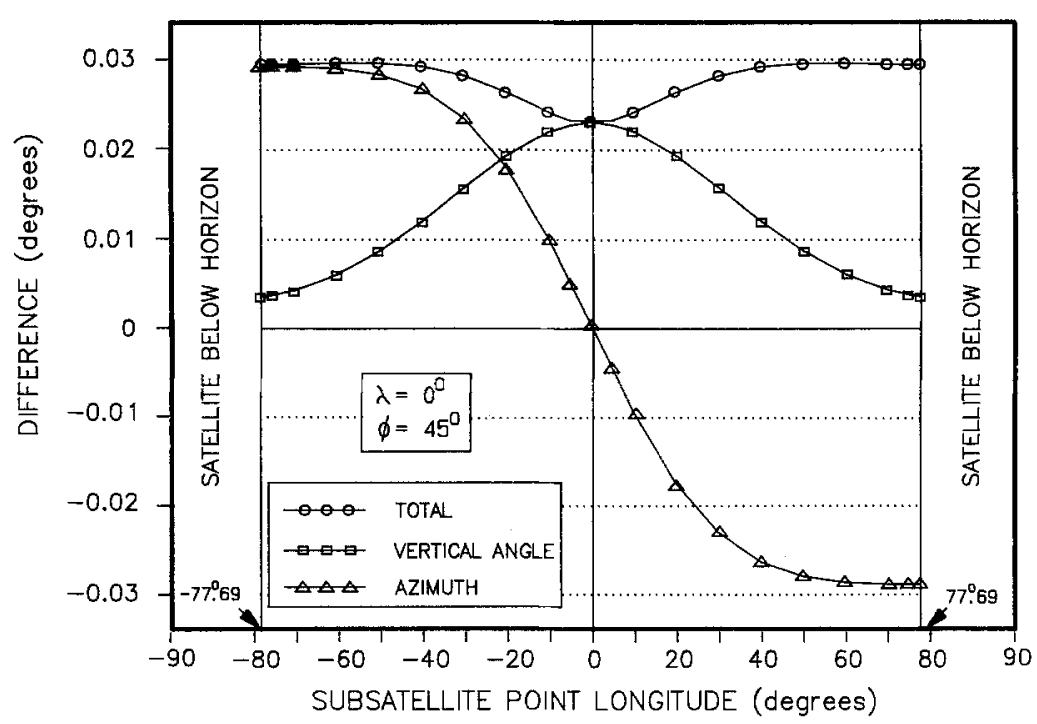

FIG. 7. Difference in Calculated Pointing Angles with Satellite and Earth Station at Different Longitudes (Ellipsoidal Method - Spherical Method)

corresponds to a minimum error in vertical angle and vice versa. More significant yet is the behavior shown by the curve representing the total or combined error. Note that when both errors in azimuth and vertical angle are taken into consideration, the total error never goes below $0^{\circ} .02$. Thus, whatever the location of the antenna and satellite, the magnitude of the total error always has a value between $0^{\circ} .02$ and $0^{\circ} .03$. As explained before, this difference between the spherical and ellipsoidal methods may not be negligible when pointing with narrow field-of-view instruments is attempted. For example, laser ranging systems to satellites require a very stringent pointing accuracy of $\pm 1^{\prime \prime}$ or $0^{\circ} .0003$ (Seeber 1993).

\section{CONCLUSIONS}

This investigation introduces a rigorous ellipsoidal alternative to determine look angles (geodetic azimuth and vertical angle) for pointing dish antennas to true geostationary satellites. The treatment is general and can be extrapolated to satellites moving in arbitrary orbits. The theory presented serves two objectives. First, it avoids reliance on spherical trigonometry, depending exclusively on basic geodetic concepts and conventional coordinate systems. Second, and most important, it assumes the earth to be an ellipsoid of revolution; thus, it is more factual than methodologies currently proposed in textbooks on satellite communications.

Results from spherical and ellipsoidal techniques are not drastically different. Nevertheless, the detected pointing discrepancies may become significant with very high gain antennas and even more crucial when electrooptical devices such as lasers are used. In conclusion, the writers regard this formulation beneficial to experts in the field of satellite communications who are not familiar with the mathematical framework of modern geodesy. There is no logical justification to pursue a spherical approximation in the 
future; the theoretical language advanced here is more coherent and provides a direct rigorous solution to the problem of look angles.

\section{APPENDIX I. REFERENCES}

Agraval, B. N. (1986). Design of geosynchronous spacecraft. Prentice-Hall, Inc., Englewood Cliffs, N.J., 101-104.

Burkholder, E. F. (1993). "Using GPS results in true 3-D coordinate systems." $J$. Surv. Engrg., 119(1), 1-21.

Ha, T. T. (1986). Digital satellite communications. Macmillan, New York, N.Y., 4144.

Leick, A. (1990). GPS satellite surveying. John Wiley and Sons, New York, N.Y., $40,195$.

Moritz, H. (1992). "Geodetic reference system 1980." Bull. Géodésique, 66(2), 187192.

Mueller, I. I. (1969). Spherical and practical astronomy as applied to geodesy. Frederik Ungar Publ. Co., New York, N.Y., 33.

Pattan, B. (1993). Satellite systems: principles and technologies. Van Nostrand Reinhold, New York, N.Y., 359.

Pratt, T., and Bostian, C. W. (1986). Satellite communications. John Wiley and Sons, New York, N.Y., 22-32.

Rapp, R. H. (1975). "Geometric Geodesy, Vols. I \& II." Dept. of Geodetic Sci. and Surv., Ohio State Univ., Columbus, Ohio.

Seeber, G. (1993). Satellite Geodesy. de Gruyter, New York, N.Y., 364.

Soler, T. (1976). "On differential transformations between Cartesian and curvilinear (geodetic) coordinates." Rep. No. 236, Dept. of Geodetic Sci., Ohio State Univ., Columbus, Ohio.

Soler, T., and Hothem, L. D. (1988). "Coordinate systems used in geodesy: basic definitions and concepts." J. Surv. Engrg., ASCE, 114(2), 84-97.

Soler, T., and Hothem, L. D. (1989). "Important parameters used in geodetic transformations." J. Surv. Engrg., ASCE, 115(4), 414-417.

\section{APPENDIX II. NOTATION}

The following symbols are used in this paper:

$(e, n, u)=$ local (right-handed) geodetic coordinate system at any point $(\lambda, \phi, h): e$-axis points to (geodetic) east; $n$ to (geodetic) north; and $u$ to (geodetic) zenith;

$H=$ orthometric height (popularly known as mean sea-level height or elevation);

$h=$ geodetic height (i.e., ellipsoidal height);

$N=$ principal radius of curvature in prime vertical plane;

$N_{g}=$ undulation (i.e., geoid height);

$v=$ geodetic vertical angle (sometimes referred as elevation or altitude);

$v^{t}=$ spherical vertical angle;

$(\mathbf{x}, \mathbf{y}, \mathbf{z})=$ conventional terrestrial reference frame (CTRF), Earth's fixed geocentric coordinate system: $\mathbf{z}$ points toward the conventional terrestrial pole (CTP); $\mathbf{x}$ passes through point of zero longitude as defined by the International Earth Rotation Service (IERS); $y$ forms right-handed coordinate system with $\mathbf{x}$ and $\mathbf{z}$;

$(x, y, z)=$ local (terrestrial) frame. Origin is at point of observation and $x, y$, and $z$ axes are, respectively, parallel to $\mathbf{x}, \mathbf{y}$, and $\mathbf{z}$ axes; 
$z=$ geodetic zenith distance;

$z^{\prime}=$ spherical zenith distance;

$\alpha=$ geodetic azimuth;

$\alpha^{\prime}=$ spherical azimuth;

$(\lambda, \phi, h)=$ curvilinear geodetic coordinates of station at point $\mathrm{P}$;

$\left(\lambda, \phi^{\prime}, R\right)=$ curvilinear spherical coordinates of station at point $\mathrm{P}^{\prime}$; and $\left(\lambda_{\mathrm{s}}, \phi_{\mathrm{s}}, h_{\mathrm{s}}\right)=$ curvilinear geodetic coordinates of satellite $\mathrm{S}$. 